Ann. Biol. anim. Bioch. Biophys., I970, 10 (I), Ior-I Io.

\title{
COMPARAISON DES MÉTHODES DE RATIONNEMENT ALIMENTAIRE SUIVANT LA NATURE DES ÉLÉMENTS ÉNERGÉTIQUES DU RÉGIME
}

\author{
B. DESMOULIN et P. DELIĆ \\ avec la collaboration technique de M. Lecourtier et P'. Peiniau \\ Station de Recherches sur l'Élevage des Porcs, \\ Centre national de Recherches zootechniques, 78-Jouy-en-Josas \\ Institut national de la Recherche agronomique
}

\section{RÉSUMÉ}

Chez le Rat blanc après le sevrage, quatre types de régimes sont comparés en associant deux taux d'huile d'arachide ( 6 ou i 8 p. Ioo) et deux taux de cellulose ( 2 ou I 2 p. roo) suivant une combinaison factorielle : $\mathrm{HC}, h \mathrm{C}, \mathrm{H} c, h c$. Ces régimes sont distribués à volonté. Par ailleurs, ils sont distribués en alimentation restreinte de façon à maîtriser les consommations soit d'énergie soit de protéines, lorsque le taux de lipides des régimes est différent. A taux de cellulose égal, l'influence du rationnement alimentaire sur la composition corporelle est étudiée sur les plans qualitatif et quantitatif.

Les conclusions sont les suivantes :

- En alimentation à volonté, la croissance et l'efficacité de la rétention azotée sont maximales : 54 à $59 \mathrm{p}$. I oo lorsque les régimes sont riches en huile. Le taux élevé de cellulose déprime de façon constante l'utilisation des nutriments énergétiques et azotés ; les rats sont plus maigres, quel que soit le taux de lipides dans le régime.

- En alimentation rationnée modérément, la restriction de protéines lorsque les régimes sont riches en huile (ajustement isocalorique) ou la restriction de calories lorsque les 'régimes sont riches en cellulose (ajustement isoprotéique) entraînent les mêmes effets : la vitesse de croissance et l'utilisation des protéines sont réduites sans permettre une réduction de l'adiposité des carcasses. L'augmentation du taux azoté des régimes restreints ne compense pas ces effets sur la croissance et accentue la chute des coefficients de rétention de l'azote : 46 à 50 p. Ioo lorsque les régimes sont riches en huile.

Quelle que soit la nature des éléments énergétiques du régime, les rats ont ajusté spontanément leur appétit pour couvrir leurs besoins énergétiques et azotés avec le maximum d'efficacité. Ils sont plus maigres en alimentation à volonté qu'en alimentation restreinte depuis le sevrage. 


\section{INTRODUCTION}

Les restrictions alimentaires, souvent préconisées pour produire des animaux maigres, ont des conséquences mal connues lorsque la nature des constituants énergétiques du régime varie et influence l'appétit de 1'animal. Les régimes riches en cellulose, consommés en plus grandes quantités, mais moins digestibles que les régimes riches en lipides, permettent généralement d'obtenir des animaux plus maigres. Chez le Rat : (Meyer, I958; Bowi,AND, I958; Henry et RÉrat, I966) et chez le Poulet : (HIIL et DANSKy, I950-I95r ; PETERSON et al., I954), les auteurs concluent que l'animal détermine librement son niveau de consommation pour couvrir en quantités équivalentes son besoin de nutriments énergétiques digestibles.

Dans ces conditions, l'excès d'adiposité des carcasses des animaux qui reçoivent les régimes riches en lipides peut être attribué à une utilisation métabolique accrue des calories digestibles. Cependant, les différences de la consommation journalière de protéines, qui sont consécutives à la "régulation énergétique " précédente de l'appétit, influencent aussi la protéinogénèse (BosshARD'T et al., I946). De cette discordance des niveaux énergétiques et azotés de la consommation, la variation de composition corporelle reste globale et peu expliquée. Il est alors utile d'imposer des restrictions alimentaires pour maîtriser les facteurs de variations observés en alimentation à volonté.

Dans l'expérience suivante, le niveau d'alimentation est imposé au Rat blanc sevré en tenant compte des différences d'appétit lorsque les régimes sont plus ou moins riches en lipides et de la différence de valeur énergétique entre ces régimes. L'alimentation égalisée peut être ainsi obtenue soit en calories soit en protéines. Par ailleurs, l'égalisation simultanée des consommations de calories et de protéines est obtenue en élevant le taux azoté du régime dont la consommation est la plus faible. Ces ajustements sont imposés par les méthodes de rationnement lorsque les régimes sont respectivement riches ou pauvres en cellulose. Le but de cette expérience synthétique est donc d'étudier l'effet des restrictions alimentaires sur l'utilisation des nutriments et la composition corporelle du Rat blanc.

\section{MATÉRIEL, E'T MÉTHODES}

\section{A. - Animaux}

Cent rats mâles de souche Wistar reçoivent pendant une semaine après le sevrage un régime préexpérimental (') équilibré et distribué à volonté. Au terme de cette période, 80 rats d'un poids moyen égal à $70 \pm 5 \mathrm{~g}$ sont retenus pour l'expérimentation. Répartis en blocs homogènes d'après le poids et le gain de poids de la semaine préexpérimentale (MCKItTrick, I947), ils sont affectés aux to traitements đu schéma expérimental décrit ci-dessous.

\section{B. - Régimes expérimentaux}

Six régimes dont la composition est donnée dans le tableau $\mathrm{I}$, sont caractérisés par un taux de cellulose Colmacel : 2 ou I 2 p. Ioo associé à un taux d'huile d'arachide : 6 ou I 8 p. Ioo. L'association factorielle des taux élevés ou faibles de cellulose et de lipides détermine quatre types de

(1) Caséine 120 , cystine 3 , amidon 527 , saccharose 200, huile d'arachide 80 , cellulose 20 , mélange salin 40, mélange vitaminique ro. 
régimes: $\mathrm{HC}, h \mathrm{C}, \mathrm{H} c$ et $h c$. La source azotée des régimes est constituée par la caséine additionnée de cystine (2,5 p. too des protéines).

- Quatre régimes présentent le même taux de I+ p. Ioo de matières azotées.

-- Deux régimes riches en lipides $(\mathrm{H})$ présentent un taux de $\mathrm{r} 6 \mathrm{p}$. Ioo de matières azotées. L'augmentation du taux de protéines de ces régimes est proportionnelle à la supériorité de la valeur énergétique des régimes $\mathrm{H}$ mesurée par rapport à celle des régimes $(h)$. Ces deux régimes sont donc riches en énergie et en protéines : l'un d'eux est riche en cellulose $(\mathrm{HC})_{a}$ l'autre est pauvre en cellulose $(\mathrm{H} c)_{a}$.

\section{TABLEAU I}

Composition des régimes, teneurs énergétiques et azotées brutes

\begin{tabular}{|c|c|c|c|c|c|c|}
\hline Constituants & $\mathrm{HC}$ & $\mathrm{hC}$ & $\mathrm{H} c$ & $h c$ & $\mathrm{HC}_{a}$ & $\mathrm{H} c_{a}$ \\
\hline Caséine .. & 16,06 & 16,06 & 16,06 & 16,06 & 18,89 & $1 \nless, 89$ \\
\hline Cystine . & 0,11 & 0,11 & 0,41 & 0,41 & 0,48 & 0,48 \\
\hline Amidon de maïs & 24,27 & 30,27 & 29,27 & 35,27 & $2 \cdot 2,82$ & $27,8:$ \\
\hline Saccharose $\ldots \ldots \ldots \ldots \ldots$ & $2 / 1,26$ & 30,26 & $29, \geq 6$ & 35,26 & 22,81 & 27,81 \\
\hline Huile d'arachide $\ldots \ldots \ldots \ldots$ & 18,00 & 6,00 & 18,00 & 6,00 & 18,00 & 18,00 \\
\hline Cellulose $\ldots \ldots \ldots \ldots \ldots \ldots$ & 12,00 & 12,00 & 2,00 & 2,00 & $1: 00$ & 2,00 \\
\hline Mélange salin $\left({ }^{1}\right) \ldots \ldots \ldots \ldots \ldots$ & 4,00 & 4,00 & 1,00 & 1,00 & 4,00 & 1,00 \\
\hline Mélange vitaminique $\left({ }^{2}\right) \ldots \ldots$ & 1,00 & 1,00 & 1,00 & 1,00 & 1,00 & 1,00 \\
\hline Teneur en matière sèche p. 100 & 93,89 & 94,06 & $y^{\prime}, 01$ & 93,83 & 93,81 & $9^{\prime}, 00$ \\
\hline Protéines $(\mathrm{g} / 100 \mathrm{~g}$ M.S.) $\ldots \ldots$ & $1,6,1$ & 14,42 & $1: 4,4$ & 14,39 & 16,70 & 16,58 \\
\hline Énergie (kcal/l $000 \mathrm{~g} \mathrm{M.S.)} \mathrm{...}$ & 5.251 & 14.511 & 5.184 & 4.510 & 5.257 & 5.227 \\
\hline
\end{tabular}

(1) Osborne et Mendel, 1918.

(2) RÉRAT et al., 1964.

\section{C. - Méthodes de rationnement - Schéma expérimental}

Dix traitements expérimentaux présentés dans le tableau 2 correspondent chacun, pour un régime défini à un niveau d'alimentation établi de la façon suivante :

TABIEAU 2

Schéma expérimental — méthodes de rationnement

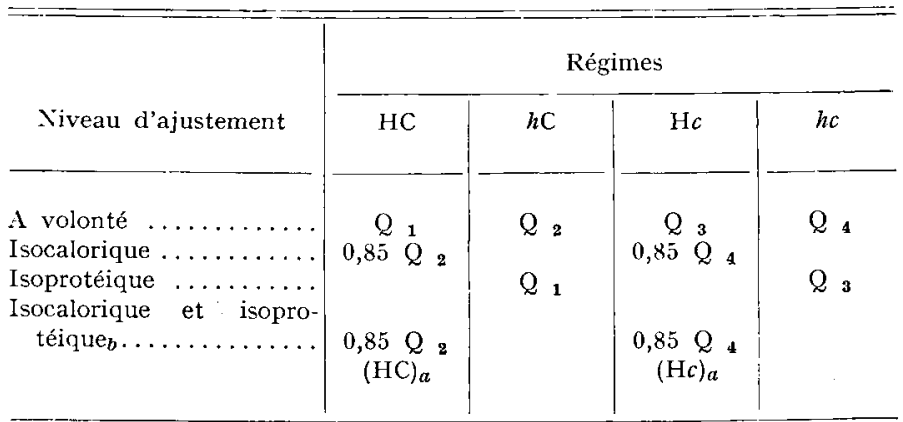

1 à 4 Quantités ingérées à volonté. 
- 4 traitements correspondent aux 4 types de régimes distribués à volonté ;

- 4 traitements correspondent aux régimes précédents distribués en quantités restreintes. Pour chaque taux respectif de cellulose $\mathrm{C}$ ou $c$, le "paired-feeding " est obtenu entre les régimes $\mathrm{H}$ et $h$ soit sur les constituants énergétiques soit sur les constituants azotés.

a) l'ajustement isocalorique s'obtient en distribuant le régime $\mathrm{HC}$ en quantité égale à $85 \mathrm{p}$. 100 de la consommation enregistrée chaque jour pour les rats qui reçoivent à volonté le régime $h \mathrm{C}$. La teneur en énergie des régimes $(\mathrm{H})$ est en effet supérieure de I 5 p. Ioo à celle des régimes $(h)$.

b) l'ajustement isoprotéique s'obtient en distribuant le régime $h \mathrm{C}$ en quantité égale à la consommation enregistrée chaque jour pour les rats qui consomment à volonté le régime $\mathrm{HC}$. faible.

Les ajustements précédents s'effectuent de la même façon lorsque le taux de cellulose est

- Deux traitements correspondent aux 2 régimes $(\mathrm{HC})_{a}$ et $(\mathrm{H} c)_{a}$ enrichis de I 5 p. Ioo en matières azotées et distribués dans les conditions identiques à celles utilisées pour l'ajustement isocalorique seul. L'élévation du taux azoté permet un ajustement isocalorique et isoprotéique entre les régimes $\mathrm{H}$ et $h$ à taux de cellulose élevé ou faible.

Dans ces conditions, l'égalisation des niveaux de consommation est qualitative car elle tient compte de la variation importante de la digestibilité des régimes (HENRY et RÉRAT, 1966).

\section{D. - Méthodes d'étude}

Pour chacun des quatre régimes distribués à volonté, les consommations journalières sont enregistrées. En alimentation restreinte, les niveaux d'alimentation sont imposés chaque jour suivant les méthodes de rationnement décrites ci-dessus. La croissance des animaux est mesurée par deux pesées hebdomadaires.

Les rats sont tous abattus à poids égal : $25^{\circ} \pm 3 \mathrm{~g}$, afin de comparer les résultats de la composition corporelle (DEsmoulin, 1967). L'analyse chimique corporelle est effectuée sur les carcasses entières homogénéisées dans un robot-coupe. Les teneurs finales en énergie et en protéines sont déterminées à partir des résultats de l'analyse chimique corporelle. Les teneurs initiales sont estimées à partir d'équation de régression des constituants corporels sur le poids vif (RERAT et al. I964). Puis les coefficients d'utilisation pratique (CUP) de l'azote et de l'énergie sont déterminés par analogie avec ceux définis par TeRroine et VAlla (I933).

$$
\begin{gathered}
\text { CUP azote }=\frac{\mathrm{N} \text { retenu dans les tissus }(\mathrm{g})}{\mathrm{N} \text { ingéré }(\mathrm{g})} \times \text { Ioo } \\
\mathrm{CUP} \text { énergie }=\frac{\text { Énergie retenue dans les tissus }(\mathrm{kcal})}{\text { Energie ingérée (kcal) }} \times \text { Ioo } \\
\text { E. }- \text { Interprétation statistique }
\end{gathered}
$$

Après analyse de variance, les moyennes sont comparées (SNEdEcor, 1956). Pour chacun des schémas factoriels $\left(2^{2}\right)$ constitué par les traitements $\mathrm{HC}, \mathrm{H} c, h \mathrm{C}$ et $h c$, l'influence des constituants énergétiques des régimes est obtenue à chaque niveau d'ajustement des consommations. Les restrictions alimentaires sont ainsi comparées sur les plans quantitatif et qualitatif.

\section{RÉSULTATS}

\section{I. - La Consommation et la Croissance}

Les résultats présentés dans les tableaux 3 et 4 , peuvent être ainsi analysés.

a) En alimentation à volonté.

L'augmentation du taux d'huile d'arachide de 6 à $\mathrm{I} 8 \mathrm{p}$. Ioo du régime, réduit de I2 p. roo en moyenne 1'appétit de 1'animal. Cependant, la consommation d'énergie reste constante à taux de cellulose égal, car les régimes $(\mathrm{H})$ présentent une valeur 
TABLEAUX 3 et 4

Conditions d'ajustement des ingérés journaliers

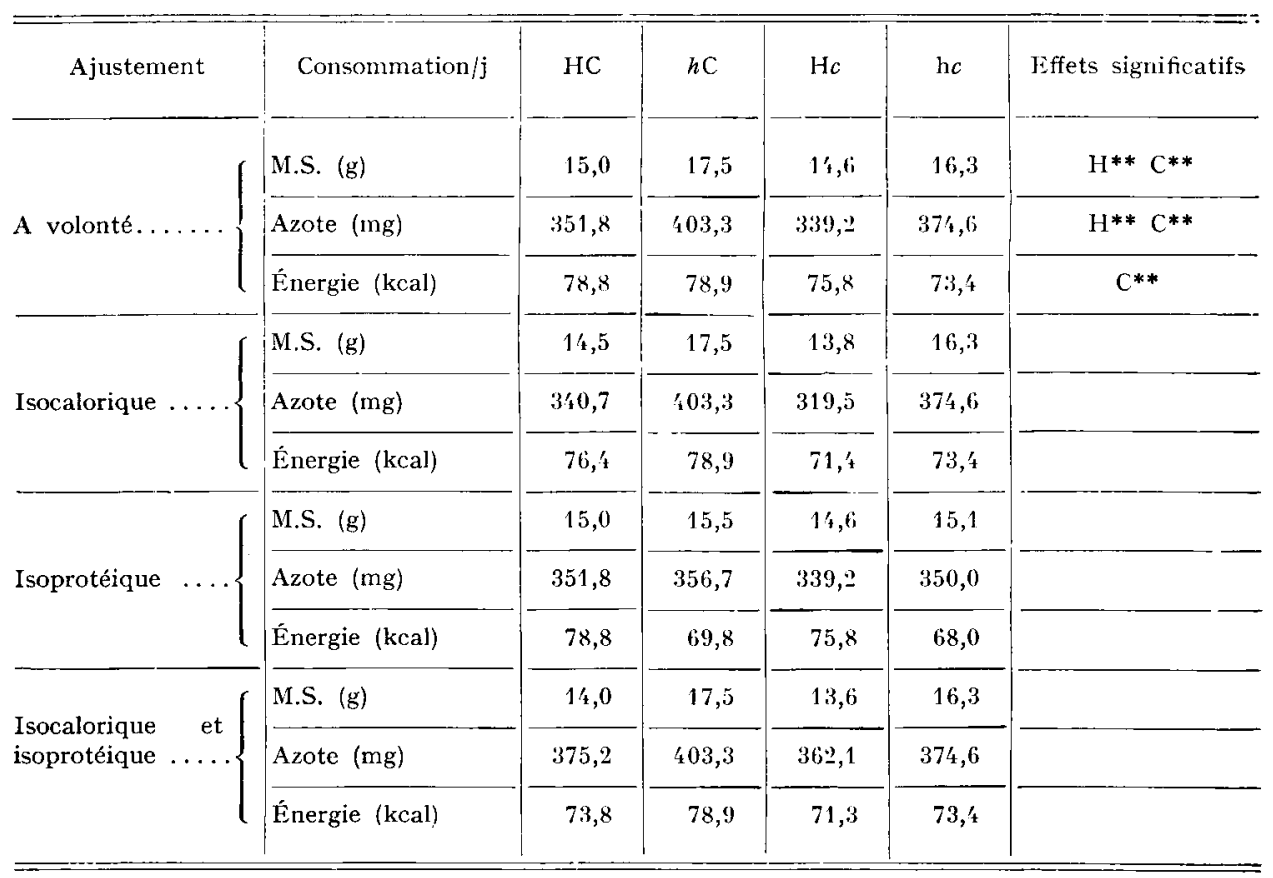

Vitesse de la croissance $(\mathrm{g} / \mathrm{j})$ et composition corporelle en protéines et en lipides (p. roo poids frais) à poids d'abattage égal (1)

\begin{tabular}{|c|c|c|c|c|c|c|}
\hline Ajustement & Régimes & $\mathrm{HC}$ & $h \mathrm{C}$ & $\mathrm{H} c$ & $h c$ & Effets significatifs \\
\hline \multirow{3}{*}{ A volonté ....... } & Gain & 6,0 & 5,7 & 6,3 & 5,6 & $\mathrm{H}^{*}$ \\
\hline & Protéines \% & 19,3 & 19,5 & 19,2 & 19,4 & NS \\
\hline & Lipides $\%$ & 12,1 & 12,4 & 14,0 & 14,0 & $C^{*}$ \\
\hline \multirow{3}{*}{ Isocalorique } & Gain & 5,4 & 5,7 & 5,6 & 5,6 & NS \\
\hline & Protéines $\%$ & 19,3 & 19,5 & 18,9 & 19,4 & NS \\
\hline & Lipides \% & 13,7 & $12,,^{\prime}$ & 17,3 & 14,0 & $\mathrm{H}^{* *} \mathrm{C}^{* *}$ \\
\hline \multirow{3}{*}{ lsoprotéique. } & Gain & 6,0 & 4,8 & 6,3 & 4,7 & $H^{* *}$ \\
\hline & Protéines \% & 19,3 & 20,0 & 19,2 & 19,3 & NS \\
\hline & Lipides \% & 12,1 & 11,7 & 14,0 & 15,9 & $C^{* *}$ \\
\hline \multirow{3}{*}{$\begin{array}{ll}\text { Isocalorique } & \text { et } \\
\text { isoprotéique } & \ldots\end{array}$} & Gain & 5,4 & 5,7 & 5,8 & 5,6 & NS \\
\hline & Protéines \% & 19,3 & 19,5 & 19,0 & 19,4 & NS \\
\hline & Lipides \% & 13,5 & 12,4 & 14,6 & 14,0 & NS \\
\hline
\end{tabular}

(1) $250 \pm 3 \mathrm{~g}$. 
énergétique brute supérieure de $\mathrm{I} 5 \mathrm{p}$. Ioo à celle des régimes (h). Malgré la réduction de leur consommation journalière de protéines, les rats soumis aux régimes $\mathrm{H}$ présentent une croissance significativement supérieure de 8 p. Ioo en moyenne à celle observée avec les régimes $h$.

L'augmentation du taux de cellulose de 2 à I 2 p. Ioo du régime accroît de 5 p. Ioo en moyenne 1'appétit de l'animal. A taux de lipides égal, les variations de croissance liées au taux de cellulose du régime ne sont pas significatives.

b) En alimentation restreinte.

Pour chaque mode d'ajustement, les résultats sont comparés à taux de cellulose égal, $\mathrm{C}$ ou $c$, dans les tableaux 3 et 4 .

- L'ajustement isocalorique seul est obtenu par défaut car la restriction alimentaire imposée aux animaux qui reçoivent les régimes $(\mathrm{H})$ est excessive. Elle reste cependant modérée par rapport à la consommation spontanée du même régime (H). En effet, le niveau de la restriction sur les protéines est accru de I2 à I6 p. Ioo relativement aux animaux qui reçoivent à volonté les régimes $(h)$. Dans ces conditions, on observe une égalisation des croissances avec les quatre types de régimes.

- L'ajustement isoprotéique et isocalorique obtenu en distribuant dans les conditions précédentes, les régimes $(\mathrm{H})_{a}$ enrichis en protéines ne permet pas de rétablir la supériorité de croissance observée en alimentation à volonté avec les régimes (H). On doit ici noter que les animaux qui reçoivent le régime enrichi en lipides, en protéines et en cellulose accumulent spontanément des refus malgré la restriction alimentaire imposée. Le régime est mieux toléré au faible taux de cellulose.

- L'ajustement isoprotéique seul, obtenu en distribuant les régimes $(h)$ au niveau de la consommation observée avec les régimes $(\mathrm{H})$ en alimentation à volonté entraîne une restriction de I7 p. Ioo de l'apport de calories. La croissance des rats soumis aux régimes $(h)$ et restreints dans ces conditions est réduite de 23 p. Ioo par rapport à celle des rats soumis aux régimes $(\mathrm{H})$. Le " paired-feeding " sur les protéines accentue donc les conséquences de la restriction énergétique sur la vitesse de croissance.

\section{La composition corporelle}

A poids d'abattage égal, les résultats rapportés dans le tableau 4 montrent que les différences de composition corporelle concernent essentiellement les quantités de lipides déposées.

a) En alimentation à volonté.

L'adiposité des carcasses est significativement plus faible lorsque le taux de cellulose est élevé. Par contre, à taux de cellulose égal, l'influence du taux d'huile d'arachide n'est pas significative.

b) En alimentation restreinte.

A l'exception du régime $h \mathrm{C}$ de faible valeur énergétique en raison d'un faible taux d'huile d'arachide et d'un taux élevé de cellulose, les restrictions alimentaires qui réduisent la vitesse de croissance, augmentent l'adiposité des carcasses à poids final égal $250 \pm 3 \mathrm{~g}$. La nature des ajustements imposés modifie ainsi les résultats 
observés en alimentation à volonté ; en effet, les différences d'adiposité dues au seul taux de cellulose peuvent être soit atténuées (ajustement isocalorique seul) soit annulées (ajustement isocalorique et isoprotéique). On peut souligner que ces résultats sont obtenus à vitesses de croissance égalisées.

\section{3. - L'utilisation pratique des nutriments énergétiques et azotés}

a) En alimentation à volonté.

L'utilisation pratique des nutriments azotés, rapportée dans le tableau 5, est supérieure de Io points en moyenne lorsque les régimes sont riches en huile $(\mathrm{H})$. A taux de cellulose égal, l'efficacité énergétique globale n'est pas influencée par le taux d'huile. Par contre, le taux de cellulose élevé (C) déprime de 3 points en moyenne l'utilisation pratique des nutriments énergétiques et azotés.

TABLEAU 5

Coefficient d'utilisation pratique (CUP) des ingérés azotés et énergétiques

\begin{tabular}{|c|c|c|c|c|c|c|}
\hline Ajustement & CUP & $\mathrm{HC}$ & $h \mathrm{C}$ & $\mathrm{H} c$ & $\mathrm{~h} c$ & Effets significatifs \\
\hline \multirow{2}{*}{ A volonté....... } & Azote & 54,1 & 45,8 & 58,8 & 48,3 & $\mathrm{H}^{* *} \mathrm{C} * *$ \\
\hline & Énergie & 17,9 & 17,5 & 21,6 & 19,9 & $\mathrm{C}^{* *}$ \\
\hline \multirow{2}{*}{ Isocalorique ..... } & Azote & 50,8 & 45,8 & 54,1 & 48,3 & $\mathrm{H}^{* *} \mathrm{C}^{* *}$ \\
\hline & Énergie & 18,2 & 17,5 & 23,5 & 19,9 & $\mathrm{H}^{* *} \mathrm{C} * *$ \\
\hline \multirow{2}{*}{ Isoprotéique. .... } & Azote & $5^{\prime}, 1$ & 45,3 & 58,8 & 42,6 & $\mathrm{H}^{* *}(\mathrm{C} \times \mathrm{H})^{* *}$ \\
\hline & Énergie & 17,9 & 16,3 & 21,6 & 19,6 & $\mathrm{H}^{* *} \mathrm{C} * *$ \\
\hline \multirow{2}{*}{$\begin{array}{l}\text { Isocalorique et } \\
\text { Isoprotéique } \ldots .\{\end{array}$} & Azote & 46,1 & 45,8 & 50,0 & 48,3 & $\mathrm{C}^{* *}$ \\
\hline & Énergie & 18,6 & 17,5 & 20,3 & 19,9 & $\mathrm{C}^{*}$ \\
\hline
\end{tabular}

b) En alimentation restreinte.

A vitesse de croissance égale, lorsque l'ajustement est isocalorique, l'efficacité azotée reste supérieure de 6 points lorsque les régimes sont riches en huile $(\mathrm{H})$. La restriction alimentaire faible a donc provoqué une dépression de 4 points du coefficient d'utilisation pratique de l'azote. En outre, l'ajustement isocalorique et isoprotéique supprime toute différence entre les régimes $(\mathrm{H})$ et $(\mathrm{h})$ concernant les CUP azotés. La consommation calorique impose le rendement optimum de l'utilisation des nutriments azotés. Au-delà de cette limite, les protéines apportées en excès sont gaspillées : elles peuvent être considérées comme un excès de nutriments dans la mesure où le rendement énergétique est accru par un dépôt de graisses supplémentaires dans la carcasse. 
Quelles que soient les méthodes de rationnement, l'influence dépressive du taux de cellulose élevé (C) est comparable en alimentation à volonté et en alimentation restreinte.

\section{DISCUSSION ETT CONCLUSION}

\section{I. - L'appétit de l'animal}

Lorsque le taux d'huile d'arachide du régime augmente de 6 à I 8 p. Ioo, les rats ajustent leur consommation de Matière sèche pour ingérer la même quantité de calories brutes. L'ajustement s'effectue à un niveau plus élevé de $5 \mathrm{p}$. Ioo lorsque le taux de cellulose est accru de 2 à I 2 p. Ioo du régime. Selon HENRY et RÉrár, I966, la digestibilité des régimes diminue de $5 \mathrm{p}$. Ioo lorsque le taux de cellulose augmente de io p. Ioo dans le régime. Nous retrouvons dans cette expérience l'égalisation des consommations de calories digestibles (Bowland, I958; SibBald, I957) par le Rat blanc avec les différents régimes consommés à volonté. Le taux azoté des régimes égal à I4 p. Ioo de protéines de caséine supplémentée en cystine, permet une couverture large du besoin azoté. Dans ces conditions, l'infuence spécifique très forte des constituants lipidiques sur la croissance et la rétention azotée s'extériorise pleinement.

L'adiposité des carcasses est plus faible lorsque le taux de cellulose est élevé, quel que soit le taux de lipides des régimes. Les niveaux de croissance permis par la consommation de nutriments énergétiques et azotés digestibles (MEYER, I958) ne permettent pas d'expliquer simplement cette infuence des constituants cellulosiques sur la composition corporelle. L'utilisation des acides gras volatils produits par les fermentations importantes dans le tube digestif des monogastriques est mal connue. Selon ÉTIENNE, (I969) les concentrations et proportions d'acides mesurées dans le gros intestin du Porc sont voisines de celles du rumen des polygastriques.

\section{2. - Les restrictions alimentaires}

La conséquence principale des ajustements effectués est la suppression de l'effet propre de l'appétit de l'animal : elle se caractérise par des restrictions de calories avec les régimes pauvres en lipides et des restrictions de protéines avec les régimes riches en lipides. Dans tous les cas, la solidarité des besoins énergétiques et azotés s'extériorise (Al.Lison, I957 ; Swanson, I95I ; Munro, I95I ; Forbes, I955 ; JACQUOT et RÉRAT, I966). La chute de la rétention azotée est particulièrement accentuée avec les régimes riches en huile. Dans ces conditions, l'équilibre qualitatif des apports alimentaires (CALLOWAY et SPECTOR, I955; LIKUSKI, I96I) est donc optimum en alimentation à volonté.

Les restrictions alimentaires perturbent l'équilibre optimum et n'ont aucun effet bénéfique pour obtenir efficacement des animaux maigres, lorsqu'elles sont modérées et appliquées dès le sevrage. Les restrictions alimentaires doivent être sévères et tardives après le sevrage pour permettre une réduction d'adiposité des carcasses (DESMOULIN, I967-I968). Chez le Rat blanc de souche Wistar, ce résultat se trouve donc généralisé lorsque la nature des éléments énergiques du régime est variable. Cette conclusion ne peut cependant pas être étendue à d'autres espèces comme le Porc, pour lequel à Ioo $\mathrm{kg}$, la restriction calorique imposée dès le sevrage entraîne une réduction d'adiposité des carcasses (Desmoulin, I969). 
Outre la différence interspécifique, les résultats obtenus sur des rats mâles sont donc différents des résultats obtenus sur des porcs mâles qui sont généralement castrés avant leur sevrage.

Rę̧u pour publication en juillet 1969.

\section{SUMMARY}

\section{A COMPARISON OF METHODS OF RATIONING FEED BY THE TYPE OF SOURCE OF ENERGY IN THE DIET}

For white rats after weaning diets with 6 or 18 per cent groundnut oil, each with 2 or 12 per cent cellulose, in factorial arrangement, $\mathrm{HC}, \mathrm{hC}, \mathrm{Hc}, \mathrm{hc}$, were compared. These diets were given to appetite. They were also given in restricted amount to regulate intake of energy or of protein when the lipid content of the diets differed. With equal cellulose contents the effect of rationing feed on body composition quantitatively and qualitatively was estimated. Conclusions were as follows :

With feeding to appetite growth and efficiency of utilization of nitrogen were maximum, 54 to 59 per cent, when the diets were rich in oil. The high cellulose depressed in a regular way the utilization of energy and nitrogen nutrients; the rats were leaner, whatever the oil content of the diet.

With moderately rationed feed restriction of protein when diets were rich in oil (adjustement to equal energy) or restriction of energy when diets were rich in cellulose (adjustement to equal protein) had the same effect : growth rate and utilization of protein were reduced without any reduction in fat in the carcass. Increase of nitrogen in the restricted feeds did not counteract these effects on growth and accentutated fall in percentage retention of nitrogen : $4^{6}$ to $5^{\circ}$ per cent when rich in oil.

Whatever the type of source of energy in the diet, the rats spontaneously adjusted their appetites to cover their energy and protein requirements with maximum efficiency. They were leaner with feeding to appetite than restricted with feeding after weaning.

\section{RÉFÉRENCES BIBLIOGRAPHIQUES}

Allison J. B., 1957. Calories and Protein Nutrition. Ann. N. Y. Acad. Sci., 69, roog-1oz4.

Bosshardt D. K., Winifred P., O'Doherty K., Barnes R. H., 1946. The influence of caloric intake on the growth utilization of dietary protein. J. Nutr., 32, 64I-65I.

Bowland J. P., Sibbald J. R., BerG R. T., Hussar N., I958. Influence of dietary fat on energy consumption and digestion and on nitrogen utilization of weanling rats. Can. J. anim.Sci, 38, 187-193.

Calloway D. H., Spector H., I955. Nitrogen utilization during caloric restriction. J. Nutr., 56, 533-544.

Desmovlin B., I967. Évolution selon l'âge et le poids de la composition corporelle du Rat blanc soumis à un rationnement énergétique après le sevrage. Ann. Biol. anim. Bioch. Biophys., 7, $28 \mathrm{I}-293$.

Desmoulin B., rg68. Influence de la durée des périodes de rationnement après le sevrage sur l'évolution de la composition corporelle du Rat blanc. Ann. Biol. anim. Bioch. Biophys., 8, 373-383.

Desmoulin B., I969. Influence de l'alimentation restreinte sur les performances du Porc : variations suivant le sexe. Journées de la Recherche Porcine en France. Paris 20-2 I février r969.

Étienne M., I969. Premiers résultats concernant les quantités d'acides gras volatils et d'acide lactique présentes dans le tube digestif du porc. Journées de la Recherche Porcine en France. Paris $20 \cdot 2 \mathrm{I}$ février I 969 .

Forbes R. M., Yohe HARTha, 1955. Effect of energy intake on the biological value of protein fed to rats. J. Nutr., 55, 499-506.

HeNRY Y., RÉRAt A., 1966. Évolution de l'ingestion spontanée de principes énergétiques en fonction de la vitesse de croissance et de la protéinogenèse chez le Rat blanc. Amino, acidespeptides, protéines. $242-262$.

Hill Ii. W., Dansky L. M., I950. Studies of the protein requirement of chicks and its relation to dietary energy level. Poultry sci., 29, 763.

Hill F. W., Dansky L. M., I95I. Influence of diet on body composition of growing chickens. Corn. Nutr. Conf. Proc., $2 \%$.

Jacquot R., Rérat A., I966. La valeur biologique des protéines. Congr. Mond. Madrid. 
Likuskr H. J., Bowland J. P., Berg R. T., rg6r. Energy digestibility and nitrogen retention by pigs and rats fed diets containing non nutritive diluents and varying protein level. Canad. J.anim. Sci., 41, 89-гог.

MeYer J. H., I956. Influence of dietary fiber on metabolic and endogenerous nitrogen excretion. $J$ Nutr., 58, 407.

Meyer J. H., I958. Interactions of dietary fiber and protein on food intake and body composition of growing Rats. Am. J. physiol., 193, 480-494.

McKirraick D. S., 1947. The selection of chicks for growth experiments and the evaluation of growth. Growth, 11, 89-99.

Osborne J.B., Mendel L. B., I9I8. The inorganic elements in nutrition. J. Biol. Chem., 34, I3I-I4o.

Peterson D. W., Grau C. R., Peek N. F., I954. Growth and food consumption in relation to dietary levels of protein and fibrous bulk. J. Nutr., 42, 24 I-257.

Rérat A., Février C., Henry Y., Lovgnon J., 1964. Évolution de la composition corporelle du Rat blanc en croissance. Ann. Biol. anim. Bioch. Biophys., 4, 35-47.

Sibbald J. R., Bowland J. P., Robble A. R., Berg R. T., I957. Apparent digestible energy and nitrogen in the food of the weanling rat. Influence on food consumption nitrogen retention and carcass composition. J. Nutr., 61, 71-86.

SNEdecor G. W., x956. Statistical methods 534 pp. Iowa State college Press, Ames.

Swanson P. P., I95I. Influence of non-protein calories on protein metabolism. Fed. Proc., 10, 660.

Terroine E. F., Valla S., 1933. Valeur comparée des différents aliments protéiques pour la crois. sance. C. R. Acad. Sci. Paris, 196, 288-290. 